\title{
Better molecular preservation of organic matter in an oxic than in a sulfidic depositional environment: evidence from Thalassiphora pelagica (Dinoflagellata, Eocene) cysts
}

Gerard J. M. Versteegh et al.

Correspondence to: Gerard J. M. Versteegh (versteeg@uni-bremen.de)

The copyright of individual parts of the supplement might differ from the CC BY 4.0 License. 


\section{Supplementary Information}

Published infrared spectra of the cysts of L. polyedrum and T. pelagica had been obtained by means of different spectrometric methods and using different brands and types of equipment. To obtain a better insight in the extent to which differences between spectra resulted from differences in cyst structure or

5 differences in measurement conditions we traced the original material and re-measured all cysts using the same analytical procedure. Supplementary figures 1 and 2 demonstrate the influence of different methods of measurement. 


\section{Lingulodinium polyedrum}

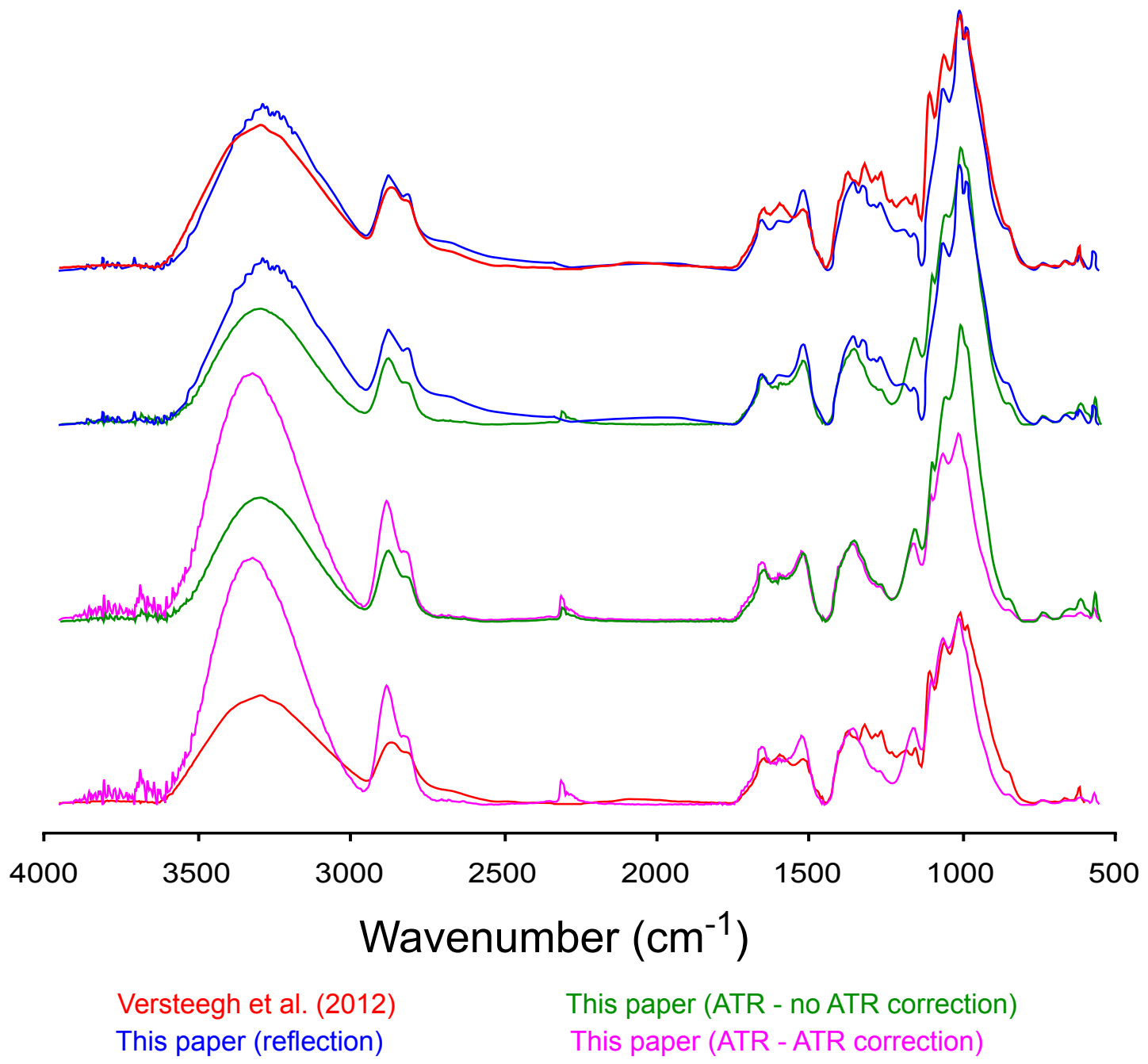

Supplementary Figure 1: Comparison of published and re-measured infrared spectra derived from culture derived Lingulodinium polyedrum cysts measured in reflection mode and micro ATR mode. All spectra are derived from same sample, and received the same sample processing. 


\section{Thalassiphora pelagica - Rhine Graben}

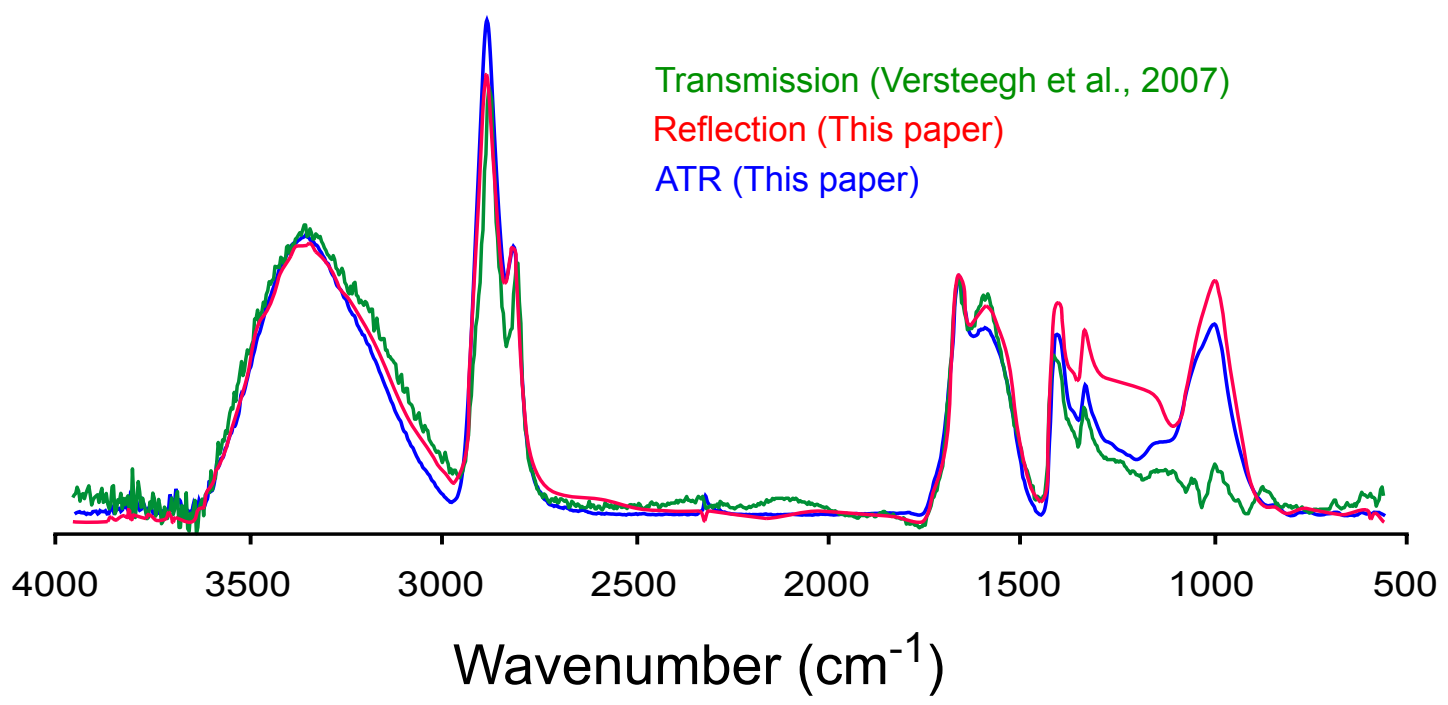

Supplementary Figure 2: Comparison of published and re-measured infrared spectra derived from Thalassiphora pelagica cysts from the Rhine Graben, measured in transmission, reflection and micro ATR modes. All spectra are derived from same sample, and received the same sample processing. 